Check for updates

Cite this: RSC Adv., 2019, 9, 15887

\title{
Towards bioinspired in vitro models of intestinal mucus
}

\author{
Lorenzo Sardelli, (D) Daniela Peneda Pacheco, (D) Anna Ziccarelli, (ID Marta Tunesi, (ID \\ Omar Caspani, Andrea Fusari, Francesco Briatico Vangosa, (D) Carmen Giordano (D) \\ and Paola Petrini $\mathbb{D}$ *
}

\begin{abstract}
Intestinal mucus is a biological structure that acts as a barrier between the external environment and the epithelium. It actively selects nutrient and drug intake, regulates the symbiosis with the intestinal microbiota and keeps the epithelium protected from the attack of pathogens. All these functions are closely connected to the chemical and structural complexity of this biological material, on which its viscoelastic and diffusive properties depend. Many models have been proposed to replicate these characteristics using glycoproteins in solution and possibly the addition of other mucus components, such as lipids and other proteins. In the field of mucus modelling, an overall view of the mucus as a material, having its own viscous, rheological and diffusive characteristics, has been undersized with respect to a pure biological-functional analysis. In this review, we propose a description of the mucus as a biomaterial, including a presentation of its chemical and structural complexity, and of its main viscoelastic-diffusive properties, in order to provide a synthesis of the characteristics necessary for the engineering of more advanced mucus models.
\end{abstract}

Received 28th March 2019

Accepted 9th May 2019

DOI: 10.1039/c9ra02368b

rsc.li/rsc-advances
The European Union has moved towards increasing Replacement, Reduction and Refining (3Rs principle) of animal studies with other scientifically validated alternatives, such as in vitro models ${ }^{10}$ as tools for high throughput screening, limiting the use of animal studies. The reliability of in vitro models is now also recognized by guidance and regulations as the European Guidance on Dermal Absorption, which defines in vitro models as mandatory assets for the analysis of percutaneous absorption of chemicals. ${ }^{\mathbf{1 4}}$ In prospective, if supported by a proper validation, they can also be proposed in substitution of in vivo models. In vitro models offer the advantage to simplify a complex phenomenon and facilitate its comprehension. The the importance of this expanding research topic. For instance, from 2008 to 2017 the European Research Council has funded 61 projects related to microbiota; more than $1 / 3$ only in the last two years. ${ }^{8}$ In fact, the authors are currently involved in the MINERVA project (EU Horizon 2020, ERC, ID 724734) that specifically studies the gut-brain-axis.

To study this intercorrelation, models that cope with the multifaceted complexity of the in vivo situation are needed. Despite of animal models being essential for medical progress, their use is often over-required. ${ }^{9,10}$ The number of replicates has to be designed to reach statistical robustness and avoid wasting resources. ${ }^{11-13}$

Department of Chemistry, Materials and Chemical Engineering "Giulio Natta", Politecnico di Milano, Milan, Italy.E-mail: paola.petrini@polimi.it

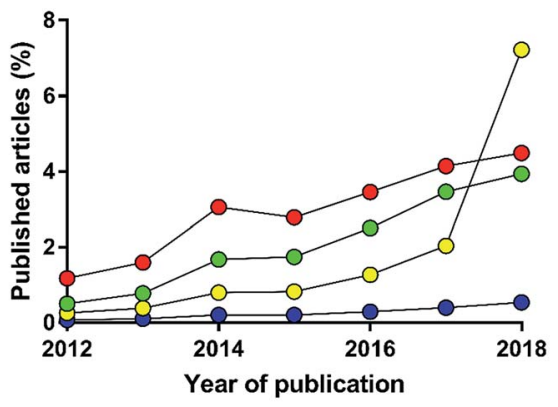

- keyword "gut" - -0 keyword "microbiome" - - keyword "gastrointestinal tract" -

Fig. 1 Percentage of manuscripts found with the keyword "gut-brainaxis" normalized to the number of manuscripts found with the keywords "gut", "microbiome", "gastrointestinal tract" or "brain" (source: PubMed). 
progressive inclusion of initially neglected features will improve its description and guide to a deeper understanding of complex phenomena.

The study in humans and animals of the inter-relation between two distinct and complex body compartments is affected by strong biases and by the extreme inter and intra species variability. Intestinal in vitro models may represent powerful tools to understand the crosstalk between gut and brain.

The importance of developing in vitro models able to replicate both the biological structure and functions of intestinal mucus is also evident when considering that the rate of failure for new drugs against inflammatory diseases within the intestinal mucosa is about $85 \% .^{15}$ To this extent, in vitro models represent physiologically relevant screening tools to select drug candidates and identify their early toxic effects. This allows not only prioritizing assets, and allocating resources, but also improve current therapies or develop new ones.

In this review, we will focus on intestinal mucus structural and compositional complexity. With a view to the increasing standardization of in vitro models required by international directives, we will present experimental data on its viscoelastic and diffusive properties. A comparative analysis of the reviewed in vivo mucus models with the physiological mucus, both from animal and human origin, is faced to provide a basis for the development of bioinspired in vitro mucus models.

\section{The relevance of the complexity of intestinal mucus}

\section{The relevance of the complexity of intestinal mucus: structural anisotropy}

In the 60's and 90's, extensive studies defined the main properties of mucus along the intestinal tract. ${ }^{\mathbf{1 6 , 1 7}}$ The intestinal mucus shows a double-layer structure (Fig. 2). The first layer is adherent and firmly attached to the epithelium, while the second, superimposed to the first one, may be collected by gently scraping the mucosa surface. ${ }^{18,19}$ The double-layer structure and the resulting gradient of mesh size and concentration of proteins, oxygen, and other chemicals are at the origin of the barrier function of intestinal mucus ${ }^{20}$ and regulates the symbiotic interactions with the microbiota. The very thin layer of mucus in the small intestine optimizes the absorption of nutrients and lower microbial population, thus decreasing the risk of infections. ${ }^{21}$ On the contrary, microbiota (more than $10^{13}$ units) grow freely in the colonic loose layer, where fermentation products like acetate, propionate and butyrate are delivered to epithelial cells. ${ }^{22}$ When genetic or external factors change the arrangement of intestinal mucus, bacteria are free to invade the epithelium, leading to severe inflammatory diseases, like Crohn's disease (Fig. 2). ${ }^{23-25}$ To prevent microbial adhesion and maintain the epithelial sterility, the layer of inner colonic mucus is thicker than in other intestinal tracts. ${ }^{26}$

The thickness is a key parameter to model intestinal mucus in vitro and it changes during disease. For instance, in the inner layer of the descending colon, mucus thickens from the healthy 134 to $232 \mu \mathrm{m}$ in Crohn's disease patients and reduces to $60 \mu \mathrm{m}$ in people suffering from ulcerative colitis. ${ }^{27}$ Unfortunately, the mucus thickness is difficult to be measured. Standard cryopreservation and fixation methods strongly modify the mucus architecture and complex post-fixation processes are needed to preserve the physiological structure. ${ }^{33}$

Structural properties like the mesh size are of primary importance in screening models for oral drugs and the development of drug carriers. These features influence the mass transport from the external environment. ${ }^{34}$ Interestingly, the attached and loose mucus layers have the same composition, but different mesh architectures. ${ }^{35}$ Proteomic analysis has shown that both layers are made up of a network of polymerized mucin2 (MUC2), but the inner layer is stratified in lamellae, while the loose one appears unfastened and unorganized. ${ }^{18,22,36}$ Latex
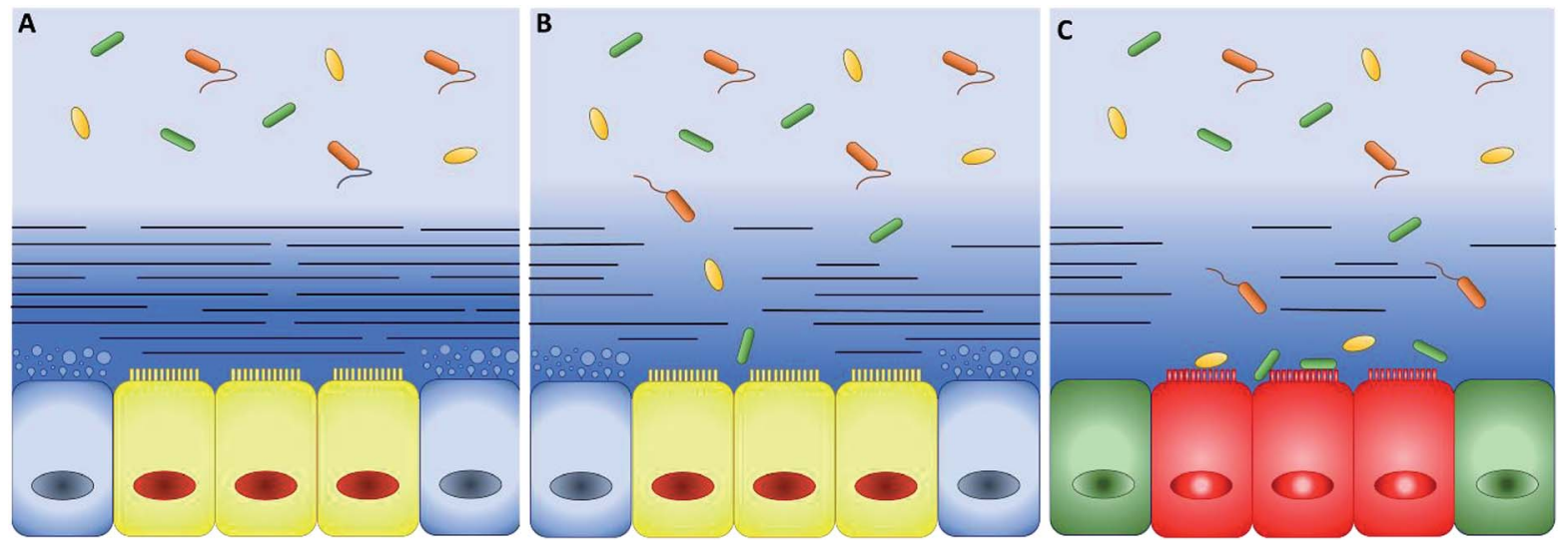

Fig. 2 (A) Schematic representation of intestinal epithelium comprising epithelial cells (yellow), mucus-secreting goblet cells (blue) and two well-defined mucus layers. The mucus has a double-layer structure: the first layer firmly attached to the mucosa (dark-blue with lines) and a loose outer layer (represented by the light-blue without lines in the top background), which may be removed easily. The thickness ratio of the attached and loose layers increases along the intestinal tract. In healthy conditions, bacteria grow only in the loose layer. (B) When the attached layer is defective or degraded by pathogens (changes in the blue shades and number of lines), epithelial cells are in direct contact with bacteria. (C) As a consequence, serious diseases such as colitis and chronic inflammation can occur (green and red colours were used to indicate a generic pathological cellular conditions). 
nanoparticles ( $500 \mathrm{~nm}$ diameter) were not able to cross the outer laye ${ }^{37}$ and atomic force microscopy (AFM) revealed a range of meshes spacing between 20 and $200 \mathrm{~nm}$, with a peak in $30 \mathrm{~nm} .^{36}$

Up to date, the mesh of the inner layer is poorly described, but it is known that the mucin network expands four times in volume when moving from the attached layer to the loose one. ${ }^{35}$ This suggests a mesh size of 5-50 $\mathrm{nm}$ and makes the attached layer responsible for diffusion impairments and epithelial defence against bacteria. ${ }^{38,39}$

\section{The relevance of the complexity of intestinal mucus: components}

Achieving compositional similarity with in vivo conditions is also a challenging task when modelling intestinal mucus in vitro. In fact, native mucus is composed of a wide range of molecules (Table 1), each one affecting the properties of the final material. ${ }^{34,40}$ Mucins are the major components and belong to the gelforming mucin (distinguished from the transmembrane mucins without structural functions). ${ }^{22}$ Even though gel-forming mucins vary in the gastrointestinal tract (e.g. MUC5A in the stomach and MUC2 in the intestine), they have the common role of structuring the mucus network, with architecture depending on the district considered. ${ }^{22}$ In particular the only gel-forming protein of intestinal mucus is MUC2, whose concentration is about $60 \mathrm{mg} \mathrm{mL}^{-1}$ in physiological conditions. ${ }^{41}$ It displays a linear structure with a variable number of tandem repeats (VNTR). They consist of sequential replicates of amino acid sequences unique to each mucin and enriched in serine, threonine, and proline amino acid residues (Fig. 3) ${ }^{18,42}$ During a complex and not completely understood polymerisation process ${ }^{42,43}$ (Fig. 4), MUC2 undergoes a sweeping increase of the molecular weight (up to $5 \mathrm{MDa}$ ) thanks to intense $O$-glycosylation rate (between $50-90 \%)^{22,35,41}$ The presence of these branched structures is a key priority for an in vitro model of intestinal mucus, as it regulates microbial adhesion and hostile activities, and provides the oligosaccharideenriched environment for microbiota metabolism. ${ }^{44-46}$ Intestinal mucus also contains lipids, cellular debris, DNA and other proteins. All these minor components are involved in the definition of its properties ${ }^{34}$ (Table 1).

Lipids heavily affect drug diffusivity and viscoelastic properties. ${ }^{28,47}$ In the absence of lipidic components, viscosity falls of $80-85 \%$, while drug diffusion increases about $90 \%{ }^{48,49}$

To describe the role of DNA on the mesh size ${ }^{41}$ of intestinal mucus and its effect on drug diffusion, ${ }^{49}$ solutions of mucin were analysed by multiple particles tracking (MPT). To mimic both adult and young native mucus, mucins derived from pigs or piglets were tested. In these models, the mesh size of mucus gels varied from $100 \mathrm{~nm}$ to $200 \mathrm{~nm}$ and DNAse treatment increased the diffusive fraction of particles (500 nm diameter) from 0.6 to $64 \%{ }^{37}$

Many proteins other than mucins are present in the intestinal mucus. ${ }^{34,50}$ Albumin, IgG and trefoil factor 3 (TFF3) peptide are the main examples. ${ }^{34,51,52}$ IgG has an important structural role, since it stabilizes MUC2 network. ${ }^{42}$ TFF3 influences the rheology of mucus by increasing tenfold the viscosity and maintaining MUC2 chains in a gel-like state with web-spider framework. ${ }^{53}$ It is also involved in the resistance against microbial activity and its role was elucidated in vitro with T84 cells (human carcinoma cell line). The cells were cultured with mucin alone or supplemented with TFF3, then treated with injurious agents. The presence of TFF3 improved the integrity of cell monolayer by $52 \%$, showing a striking cooperative interaction between glycoproteins and TFF3. ${ }^{54}$ Actually, the resistance against bacteria requires the contribution of an impressive number of peptides. ${ }^{55}$ Most of them are secreted by Paneth cells ${ }^{51}$ and, interestingly, remain close to the epithelium without spreading to the lumen. ${ }^{56}$ This behaviour clearly remarks the different properties of the mucus layers: the inner attached layer is a defensive barrier against bacteria, while the outer loose layer hosts a wide microbial niche. ${ }^{38,39}$ Despite the fundamental role that structural anisotropy and chemical complexity play in intestinal mucus functions, they have been poorly integrated in in vitro mucus models, especially in the field of drug delivery. ${ }^{57}$ This is not a limit to underestimate. In a recent study, a bio-engineered artificial mucus (made of polysaccharide hydrogel) greatly impacted the diffusion of polystyrene (PS) nanoparticles when inserted in standard laboratory equipment for permeability measurements. ${ }^{58}$

\section{The intestinal mucus models}

In vivo and ex vivo mucus models. Intestinal mucus has at least two functions: it guarantees a perfect physical separation between bacteria and cells and it regulates the absorption of substances from the lumen, acting as a selective membrane. ${ }^{59} \mathrm{In}$ vitro models should display a thickness and a ratio between the thickness of the loose and attached layers comparable to native mucus, as these parameters are involved in the specific functions of the different intestinal tracts. ${ }^{41}$

Table 1 Composition $(\% \mathrm{w} / \mathrm{v})$ of intestinal mucus according to literature ${ }^{a}$

\begin{tabular}{|c|c|c|c|c|c|c|c|}
\hline Species & Tract & Water & Proteins & Mucins & Lipids & DNA & Reference \\
\hline Rat & Small intestine & 84 & 8.2 & & 2.64 & $* *$ & 28 \\
\hline Rat & Small intestine & 84 & 7.2 & 5.8 & $* *$ & 0.2 & 29 \\
\hline Pig & Duodenum & $* *$ & $* *$ & 4 & $* *$ & $* *$ & 31 \\
\hline Pig & Colon & $* *$ & $* *$ & 3 & $* *$ & $* *$ & 31 \\
\hline Human & Colon & 82 & $* *$ & 6.63 & $* *$ & 0.19 & 32 \\
\hline
\end{tabular}

$a * *$ Data not available. 


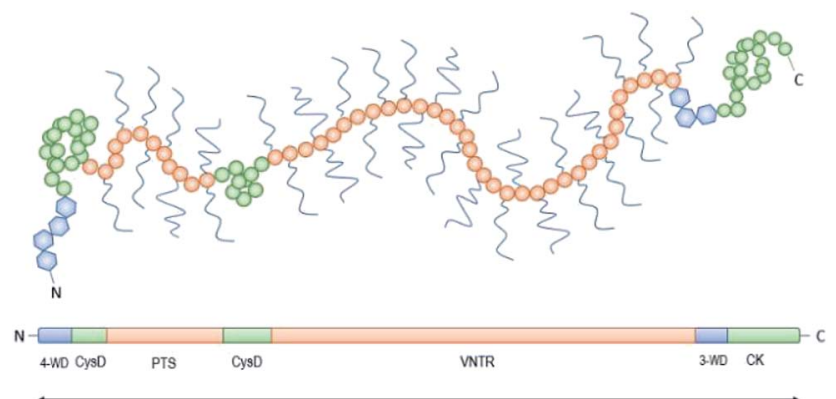

Fig. 3 Schematic representation of the structure of mucin-2 (MUC2): the $\mathrm{N}$-terminus is followed by four von Willebrand domains (4-WD, blue hexagons) and two rich cysteine domains (CysD, green circles) with numerous disulphide bonds. The central region shows the first Ser/Thr/Pro rich 21 repetitive region with an irregular amino acid sequence (PTS, pink circles), and the second regular tandemly repeated motifs of 23 amino acids with variable number of tandem repeats from 51 to 115 (VNTR, pink circles). The Ser/Thr/Pro rich series form two highly glycosylated regions upstream the C-terminus, with three von Willebrand domains (3-WD, blue hexagons) and the cysteine knot (CK).

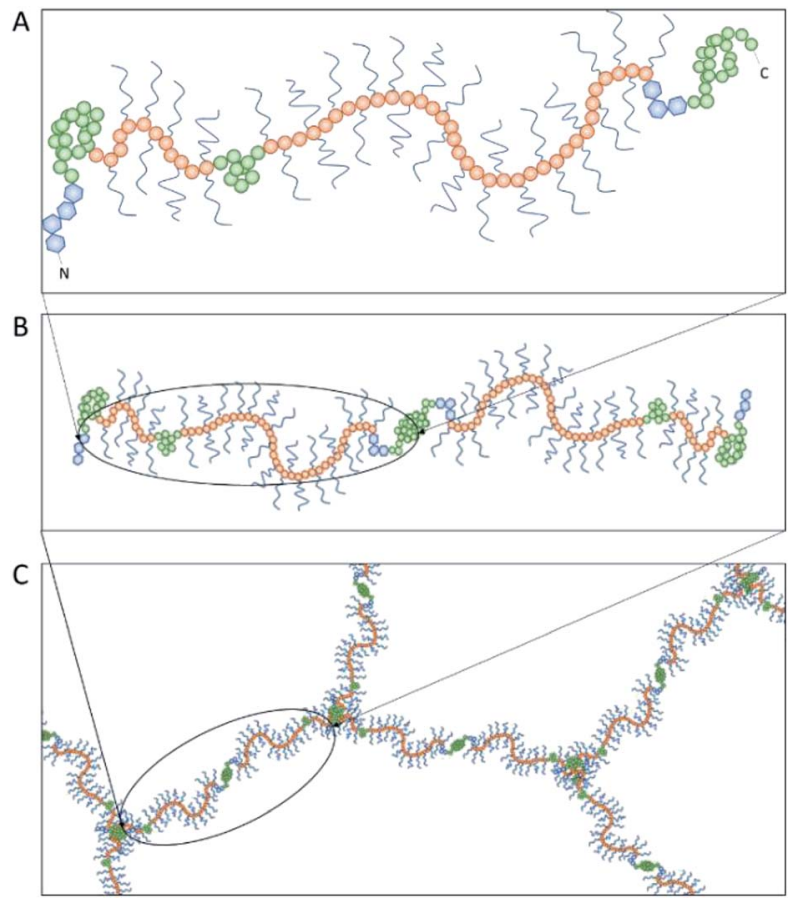

Fig. 4 The polymerization of mucin-2 (MUC2) takes place into the endoplasmic reticulum of goblet cells, where MUC2 monomers (A) dimerize at the cysteine knot ( $\mathrm{CK}$, see Fig. 3) by disulphide bonds (B). After dimer formation, MUC2 is transported to the Golgi apparatus and then secreted in the extracellular environment. Here, it undergoes trimerization when the four von Willebrand domains of three $\mathrm{N}$-termini are connected by sulphide bonds, appearing in a trefoil-like shape (C).

To elucidate the role of the mucus in drug delivery and symbiosis with bacteria, different models have been used ${ }^{60}$ (Fig. 5).

The gold standard for studies related to human intestinal permeability is the Loc-I-Gut ${ }^{\mathrm{TM}}$ model. It allows for in vivo acquisition and it consists of an oral perfusion system made up of a multichannel tube with two balloons. ${ }^{61,62}$ Similarly, in vivo studies on rat models are also performed with single-pass perfusion: the gastrointestinal tract under investigation is isolated and cannulated with plastic tubing, avoiding disturbance in the blood flow and thermal shock.

The model based on the everted intestine is the only ex vivo model proposed for intestinal mucus. It was conceived in 1985, improved in 1993 and then maintained basically unchanged. ${ }^{69,70}$ The selected intestinal area is excised and cut in several rings. Each ring is twisted (so that the mucosa faces the external environment), sutured at one edge, filled with saline and closed at the other extreme. The samples are transferred to an incubation flask containing the drug and sampling may be done at different time points. ${ }^{70}$

\section{In vitro mucus models}

Cellular mucus models. To overcome the limitations of these in vivo and ex vivo models (e.g. low repeatability, high costs, and animal sacrifices), several in vitro models have been proposed. ${ }^{62,71}$ Caco-2 cells are commonly used because of their similarity in morphology and functionality with enterocytes; however, they are not mucus-secreting cells. ${ }^{72,73}$ This is why mucus-secreting cells like HT29-MTX cells are used as an alternative to (or co-cultured with) Caco-2 cells. ${ }^{74-76}$ The triple co-culture of HT29-MTX, Caco-2 and RaijB cells represents a further improvement, thanks to the ability of RaijB cells to induce the differentiation of Caco- 2 cells into M-cell, that are involved in defence mechanisms. ${ }^{77-79}$

Advanced culture systems mimicking native threedimensional microenvironment and providing a dynamic flow of medium reduce the differences between in vitro models and physiological conditions, for instance in terms of integrity of the tight junctions across the cell monolayer (evaluated by transepithelial electrical resistance, TEER) and permeability. However, cellular models have intrinsic limitations that are difficult to overcome. For example, they are not able to replicate the hierarchical structure of the mucosa, except in extremely complex environments. ${ }^{80-82}$ Furthermore, the HT29-MTX goblet cell line secrete the gastric MUCA5 protein and not the intestinal MUC2, leading to the presence of a mucus with properties differing from physiological conditions. ${ }^{\mathbf{8 3} 84} \mathrm{Up}$ to date, in vitro models of intestinal mucus include physiological mucus models, mono-component mucus models, and multicomponent mucus models. This section will focus on artificial mucus models and provide a critic comparison of their physicochemical and viscoelastic properties with respect to physiological mucus.

Physiological mucus models. Clearly, the human physiological mucus is the most representative sample, though limited availability, lack of volunteers, difficulty to obtain high volumes and high inter/intra-variability make it difficult to isolate/use.

A large amount of data is available from different animals. Mucus is isolated from the gastrointestinal mucosa of large animals like pigs and directly used (fresh mucus) or purified before reconstruction (mucin solutions or gels). ${ }^{\mathbf{4 0 5} 85}$ However, high intra-animal variability of important parameters, like 


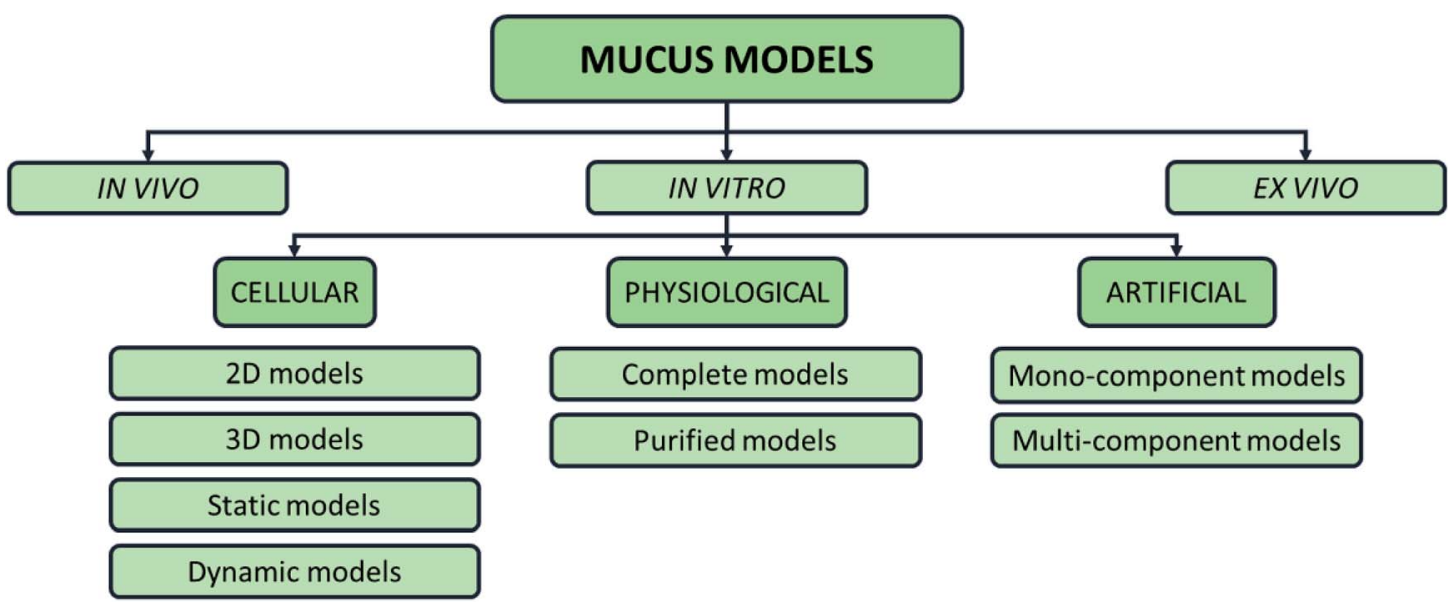

Fig. 5 Possible classification of intestinal mucus models proposed in the literature, including in vitro, in vivo, and ex vivo models

mucin concentration, greatly reduces the reliability of these models to reproduce human mucus. ${ }^{86,87}$ Moreover, the viscoelastic properties of physiological samples are strictly dependent on the conservation method of the mucus. For example, mucus samples scraped from the small intestine of pigs shows a shortening of their linear viscoelastic region (LVR) of the 70\% if incubated at $37^{\circ} \mathrm{C}$ for $30 \mathrm{~min}$ before use. ${ }^{88}$

Physiological mucus models include all the mucus components like lipids, proteins, DNA and cell debris, that contribute to mucus properties, such as mesh size, viscoelastic behaviour and net charge. ${ }^{89,90}$ For instance, samples of physiological mucus scraped from the jejunum of pigs and piglets show a reduction of their LVR extension and storage modulus $\left(G^{\prime}\right)$ values at any strain and a decrease of viscosity at a given shear rate when treated with DNAse. ${ }^{37}$ These observations are corroborated by the evidence that in the airway mucus, the presence of DNA from neutrophils during inflammation leads to increased viscosity, which may contribute to the pathophysiology of severe disease like cystic fibrosis. ${ }^{91}$

The rheology of physiological mucus is strictly dependent on the age of the animals and characterised by wide variability between subjects and the intestinal tract of interest (Table 2). ${ }^{40,92}$ Indeed, viscoelastic parameters like storage $\left(G^{\prime}\right)$ and loss moduli $\left(G^{\prime \prime}\right)$ take different values depending on the analysed intestinal section, while maintaining not only the typical characteristics of a gel $\left(G^{\prime}>G^{\prime \prime}\right)$, but also similar viscosity (Fig. 6). The storage modulus, as the thickness of the mucus, increases along the intestinal tract, being minimum in the proximal intestine, maximum in colon and intermediate in the jejunum. Physiological and purified mucus models are commonly used in vitro to study pharmacological diffusion, nanoparticle motions and rheological characterisation, as summarized in the following paragraphs.

\section{Artificial mucus models}

Mono-component mucus models. In vitro intestinal monocomponent mucus models are made only of mucins either in solution or reconstituted. Easily available and standardized, commercial mucins from pigs (PGM) or bovines (BSM) overcome the high intra- and inter-variability of physiological mucus but lack its diversified chemical composition (Table 3). Apart from mucins, physiological mucus comprises many other important components ${ }^{89}$ that are lost during the extraction and purification process. ${ }^{93,94}$ Moreover, solutions of purified mucins do not reproduce accurately the viscoelastic properties of physiological mucus and show different diffusion coefficients. ${ }^{30,95}$ This is possibly due to a breakdown of the disulphide bonds in the dimers and trimers of mucins treated with pepsin for the commercialisation. ${ }^{87}$

The differences in drug diffusion between physiological intestinal mucus and mono-component mucus models were investigated by analysing self-diffusion coefficients of different drugs (that is the diffusion coefficient multiplied by the molecular weight). ${ }^{30,49}$

The absence of the other chemical components of mucus, like lipids, in the mono-component model results in facilitated drug diffusion. Hydrophilic or hydrophobic molecules may pass through the mucus similarly, suggesting that mucin alone is not sufficient to replicate the selective barrier properties of physiological mucus. When the medium was changed from monocomponent mucus model to physiological mucus, the diffusivity of propranolol and testosterone decreased by $25 \%$, which was also observed for hydrocortisone and metoprolol with a $50 \%$ decreased diffusivity ${ }^{49}$ (Fig. 7). The decreased diffusion of lipophilic drugs like hydrocortisone and testosterone may be explained by the chemical interaction with the lipids in physiological mucus. When they are not included in the model, the

Table 2 Main models of physiological mucus proposed in the literature

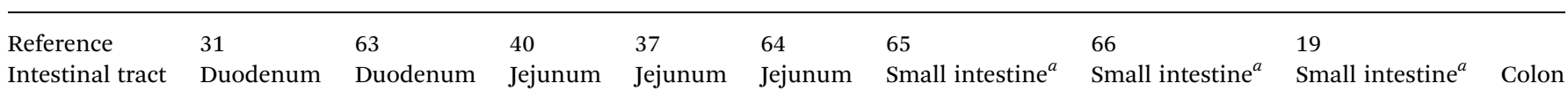

${ }^{a}$ No information is specified on the intestinal tract. 
A

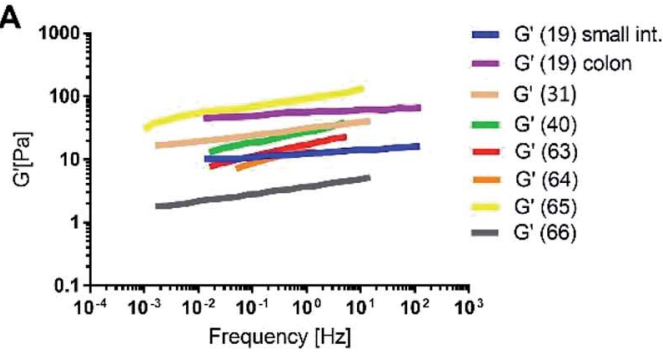

C

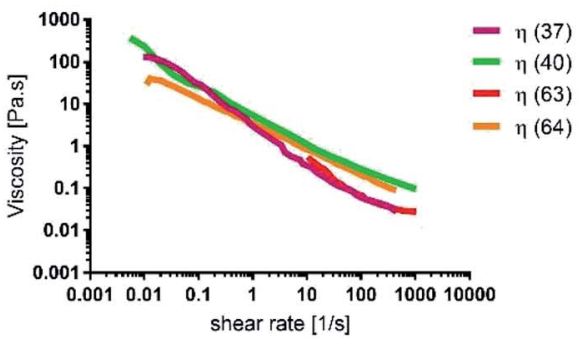

B

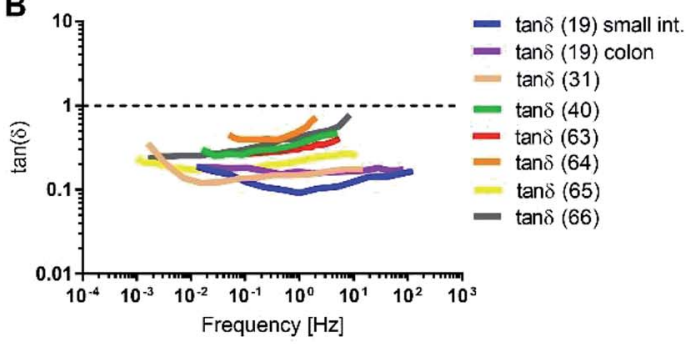

D

\begin{tabular}{|c|c|c|c|c|c|c|c|c|}
\hline REFERENCE & (19) & (37) & (40) & (31) & (63) & (64) & (65) & (66) \\
\hline TEMPERATURE $\left({ }^{\circ} \mathrm{C}\right)$ & $25^{\circ}$ & $37^{\circ}$ & $37^{\circ}$ & $25^{\circ}$ & $25^{\circ}$ & $37^{\circ}$ & $25^{\circ}$ & ** \\
\hline
\end{tabular}

Fig. 6 (A) Storage modulus, (B) $\tan \delta$, (C) shear viscosity for intestinal physiological mucus samples and (D) the temperature of the rheological test. The data were digitally extracted from the different sources. Each model is named after the reference number (numbers in brackets) and the type of sample. Before plotting, the data were homogenized in units of measurement to make them comparable. **Data not available.

diffusion is subsequently overestimated, as was also observed in very diluted $(1.5 \% \mathrm{w} / \mathrm{v})$ solutions of physiological mucus. Polystyrene nanoparticles modified with amino, carboxylic $\left(\mathrm{COOH}^{-}\right.$ PS) and sulphate groups - to render the surfaces either positively or negatively charged - were used to observe differences in nanoparticles motion measured with the mean square displacement (MSD) inside physiological and mono-component mucus models. ${ }^{68}$ Positively charged nanoparticles had low MSDs due to the interactions with mucins in both monocomponent and physiological mucus models, while negatively charged nanoparticles, receded by the glycoprotein charges, were able to move freely. The diffusion time needed for positively charged nanoparticles to reach the same diffusivity passed from 0.1 to $10 \mathrm{~s}$ in physiological and mono-component models, respectively. Moreover, the number of diffusive particles modified with sulphate groups and carboxylic groups doubled and tripled through mono-component model and physiological mucus, respectively. These results suggest that the presence of a mesh in the mucus, not present in the monocomponent mucus model, assume an extremely important role in the diffusive processes, as it may entrap and/or deviate nanoparticles. This hypothesis is also corroborated considering that the diffusivity of carboxylated nanoparticles with comparable size was doubled (from 0.14 to $0.30 \mu \mathrm{m}^{2} \mathrm{~s}^{-1}$ ) in another purified mucus model with the same mucin content $(3 \% \mathrm{w} / \mathrm{v})$, but at neutral $\mathrm{pH}^{67}$ The low $\mathrm{pH}$ of the mucus model promotes not only a gel-like transition of the mucins in solutions, but also increased viscosity that subsequently decreased nanoparticles mobility. At neutral $\mathrm{pH}$, mucins are not crosslinked, and present lower viscosity. Hence, the nanoparticles diffuse more freely in every direction, ${ }^{95}$ as demonstrated by time-diffusivity measurements using a dynamic light scattering (DLS) method. ${ }^{95}$ The different diffusion of nanoparticles in the two models (both at neutral and acid $\mathrm{pH}$ ) is strongly related to the poor compositional similarity with mucus. The intestinal mucus is a biological gel where the elastic contribution $\left(G^{\prime}\right)$ exceeds the viscous one $\left(G^{\prime \prime}\right)$ in small amplitude oscillating shear test (Fig. 6). Solutions of reconstituted mucin from the intestinal tract of interest preserve a solid-like behaviour $(\tan \delta$ $=G^{\prime} / G^{\prime \prime}<1$ ), under a small amplitude oscillating shear test, if the concentration of mucin is sufficiently high (close to $2 \% \mathrm{w} / \mathrm{v}$ ) (Fig. 8B).$^{63}$ Below this threshold, the mono-component models show a $\tan \delta$ slightly higher than one, suggesting a viscous contribution to the rheological behaviour higher than the elastic one. Under constant strain rate tests, this is reflected in a lower steady state viscosity at any strain rate for monocomponent mucus models with respect to the physiological mucus, even if the shear thinning behaviour is preserved (Fig. 8C). ${ }^{40,63}$

Like physiological mucus, mono-component mucus models based on mucins from different portions of the gastrointestinal tract display different viscoelastic properties even if at the same concentration. For instance, the mono-component mucus model composed of $4 \%(\mathrm{w} / \mathrm{v})$ duodenal mucins shows a storage modulus that is ten times higher than the one of $4 \%(\mathrm{w} / \mathrm{v})$ jejunal mucins. ${ }^{31,63}$ This is a considerable limitation of mucin purification from isolated physiological mucus. It may be overcome by using commercial mucins, that in spite of altered structure exhibit less lot-to-lot variability and are available in greater amounts.

Thanks to commercially available mucins, mono-component mucus models can provide versatility and repeatability, although they imply a deviation from the physiological behaviour.

It should be noted that the rheological tests on monocomponent models are not always performed under comparable conditions in terms of oscillatory frequency and shear rate. Moreover, the different temperature used in the experimental set up are also different. As the viscoelastic properties of 


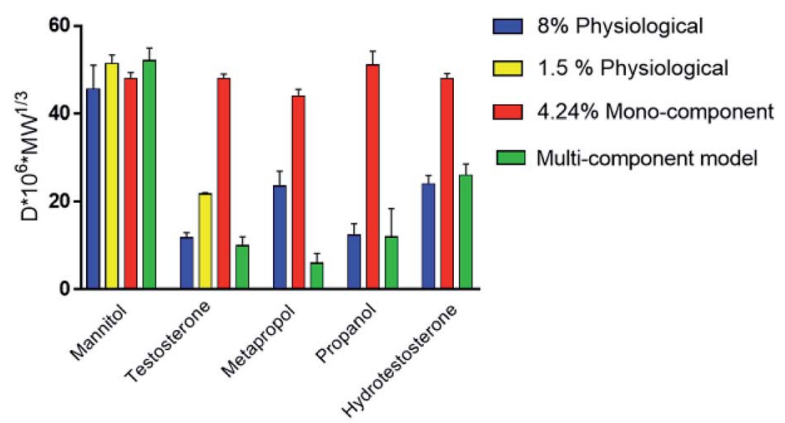

Fig. 7 Self-diffusion coefficients of drugs in the medium of monocomponent and multi-component mucus models. The data were digitally extracted from the different sources. Each model is named after the type of sample. Before plotting, the data were homogenized in units of measurement to make them comparable.

biological tissue are influenced by the temperature, ${ }^{97}$ the variability introduces defect in the consistency of these comparisons.

Comparing the different models and establishing the most suitable for a certain intestinal tract is difficult. Nevertheless, rheological tests help in evaluating per se if current monocomponent mucus models are suitable to mimic the intestinal viscoelastic properties by varying mucin concentration and source.

The advantages of moving from a complete model like the physiological one to an incomplete one like the monocomponent model might seem dubious. Undoubtedly, the physiological model allows analysing the mucus in its entirety, but it clouds the contribution of each component over the final properties. Instead, other mucus components may be added one by one to a model made up by mucins only, identifying the contribution of each component on the properties of the complete, reconstituted, model. Mono-component mucus models may therefore be considered as the natural starting point for more complete multi-component mucus models.

Multi-component mucus models. Multi-component mucus models have been recently proposed to overcome the limits of mono-component mucus models in terms of chemical composition and structure. These models combine a physiological concentration of mucin (reconstituted or in solution) with other mucus components like lipids, proteins or DNA (Table 4). With reference to rheology, multi-component mucus models based on mucins in solutions, under small amplitude oscillation in shear, display a viscoelastic behaviour with comparable values of $G^{\prime}$ and $G^{\prime \prime}$ and, as a consequence, $\tan \delta \cong 1$ (ref. 96) (Fig. 9A and $\mathrm{B}$ ).

A multi-component mucus model made up of $0.9 \%(\mathrm{w} / \mathrm{v})$ polyacrylic acid (PAA) as the gelling agent, exhibited $\tan \delta<1$ and $G^{\prime}$ values similar to physiological mucus. The agreement with physiological mucus was especially appreciable at low frequencies $(<1 \mathrm{~Hz})$, corresponding to the physiological intestinal contraction, note as migrating motor complex. ${ }^{98,99}$

As for the steady state response to constant shear rate test, multicomponent model with $0.9 \%$ PAA exhibited a shear thinning behaviour comparable to that of physiological model, but with a viscosity at any shear rate higher than that of 
A

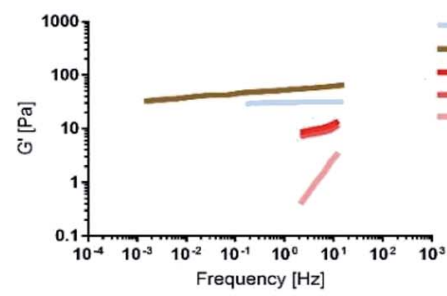

C

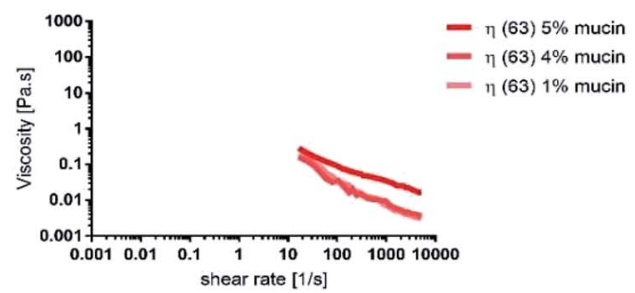

B

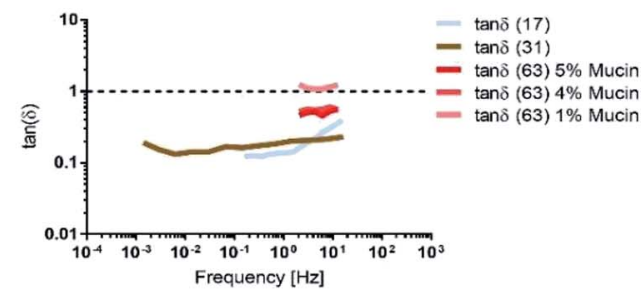

D

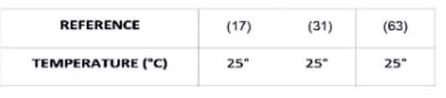

Fig. 8 (A) Storage modulus, (B) $\tan \delta,(C)$ shear viscosity for intestinal mono-component mucus models and (D) the temperature of the rheological test. The data were digitally extracted from the different sources. Each model is named after the reference number (numbers in brackets) and the type of sample. Before plotting, the data were homogenized in units of measurement to make them comparable.

Table 4 Multi-component mucus models proposed in the literature

\begin{tabular}{|c|c|c|c|c|}
\hline \multirow[b]{2}{*}{ Reference } & \multirow[t]{2}{*}{ Chemicals (\% w/v) } & \multicolumn{3}{|c|}{ Multi-component mucus model } \\
\hline & & 40 & 49 & 96 \\
\hline Glycoproteins & Mucin & 5 & 0.424 & 6 \\
\hline \multirow[t]{3}{*}{ Lipids } & Linoleic acid & 0.11 & 2.6 & \\
\hline & Colesterol & 0.36 & 0.38 & \\
\hline & Phosphatidylcholine & 0.18 & 0.19 & \\
\hline & Pig serum albumin & & 3.3 & \\
\hline DNA & & & 5.3 & \\
\hline Gelificant & Polyacrylic acid & $0.3-0.9$ & & \\
\hline \multirow[t]{2}{*}{ Solution } & Buffer & Hepes & Phosphate & Hepes \\
\hline & $\mathrm{pH}$ & 7.4 & $6.4-6.9$ & 7.4 \\
\hline
\end{tabular}

physiological mucus. When the content of PAA was reduced to $0.3 \%(\mathrm{w} / \mathrm{v})$, the shear viscosity decreased and better approximated the viscosity of pig physiological mucus (Fig. 9C).

The differences between the viscoelastic properties of the multi-component mucus model in solution and in gel may be explained not only by the presence of the PAA, but also because of the lipid content $(0.32 \%$ in solution and $0.65 \%$ in gel).

One of the main prerequisites for in vitro modelling is the versatility of the system. In this view, the multi-component model in gel is more suitable to mimic the intestinal mucus, as it allows changing component concentration to match the different rheological features of physiological mucus, like steady state viscosity, $G^{\prime}$ and $\tan \delta$. For instance, by reducing the concentration of PAA, it is possible to reproduce the native shear thinning behaviour, physiologically relevant for mucus clearance and microbial location and motion. On the opposite, the multi-component model with $0.9 \%(\mathrm{w} / \mathrm{v})$ PAA reproduces the mechanical response of physiological mucus at low frequencies (that is under the stimulus of the migrating motor complex).

When compared to mono-component mucus models, the diffusivity of charged and neutral drugs in multi-component mucus models is similar to physiological mucus (Fig. 7). In particular, lipids revealed to be the most influencing components for drug-mucus interaction. Indeed, the diffusivity of mannitol and testosterone were similar in both multicomponent mucus model and specific lipid mixture, ${ }^{\mathbf{4 9}}$ suggesting that their contribution is sufficient to explain the difference between the models and physiological conditions. Their importance was further highlighted by considering that protein content, comparable with the lipidic one (3.83 vs. 3.60\% $\mathrm{w} / \mathrm{v}$, respectively), had minimal influence on drug diffusion; in 
A
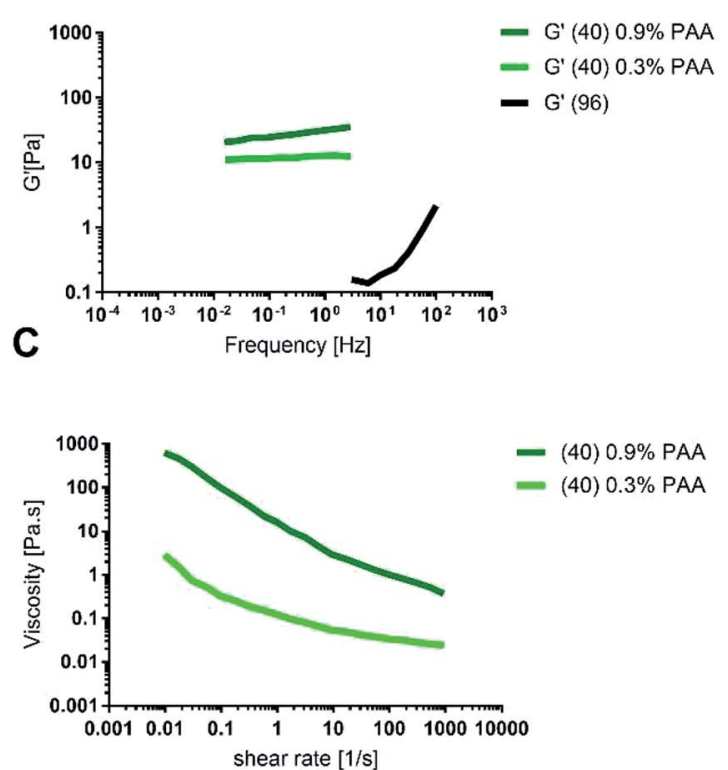

B

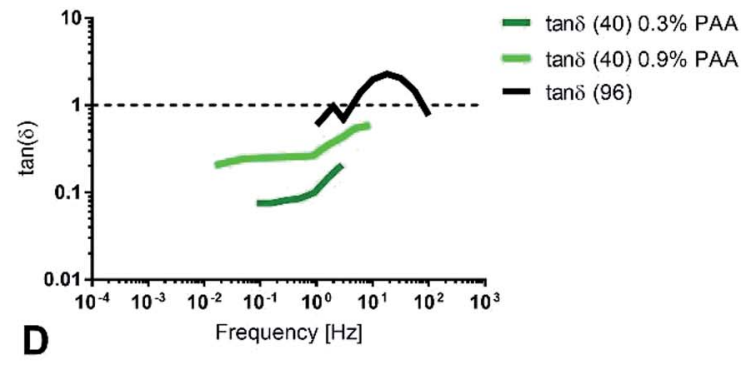

Fig. 9 (A) Storage modulus, (B) $\tan \delta$, (C) shear viscosity for intestinal multi-component mucus models and (D) the temperature of the rheological test. The data were digitally extracted from the different sources. Each model is named after the reference number (numbers in brackets) and the type of sample. Before plotting, the data were homogenized in units of measurement to make them comparable.

other words, proteins alone cannot explain the dissimilarities between the model and the physiological mucus.

Pharmacological investigation performed on a PAA-based multi-component model ${ }^{40}$ showed different results. Although the low concentration of lipids $(0.65 \% \mathrm{w} / \mathrm{v})$ was sufficient to recapitulate the barrier properties of mucus against lipophilic drugs, the permeability of mannitol $\left(P=0.131 \times 10^{-2} \mu \mathrm{m} \mathrm{s}^{-1}\right)$ was 10 times less than that of testosterone $\left(P=1.065 \times 10^{-2} \mu \mathrm{m}\right.$ $\mathrm{s}^{-1}$ ), with a lag time of $46 \mathrm{~min}$. Oppositely, the diffusion of testosterone was immediate. The disagreement of these data with other mono and multi-component mucus models could be explained by the presence of PAA $(0.9 \% \mathrm{w} / \mathrm{v})$, its interaction with the OH-groups of mannitol and the consequent reduction in drug permeability.

A comparison of the diffusion performance among the artificial mucus models is more difficult, since works analysing nanoparticle diffusivity adopt different tests and parameters (Fig. 10).

Fluorescent PS nanoparticles (diameter: $200 \mathrm{~nm}$ ) were functionalized with carboxylic groups and used to model a carrier system. Particle diffusivity was studied in both physiological condition (mucus from the small intestine of pigs $^{68}$ ) and multi-component mucus models. ${ }^{96}$ Particle trajectories were used to calculate time-averaged MSDs, that were subsequently applied to determine time-dependent diffusion coefficients and characterized the transport models of individual particles. The ensemble MSDs of carboxylate-modified particles, both in the multi-component model and the physiological mucus, showed a non-linear dependence on time, that is the expected behaviour for the diffusion of nanoparticles through the non-homogeneous structure of mucus (Fig. 10).
The differences between the values recorded in physiological mucus and multi-component mucus model in solution could be attributed to the different tools and methods adopted during the experiments. Their properties were acquired with a different spatial resolution (50 and $5 \mathrm{~nm}$ ). The particle solution $\left(0.00125 \mathrm{mg} \mathrm{mL} \mathrm{m}^{-1}\right)$ in physiological mucus was deposited on top and analysed only after 2 hours, while in the multicomponent model a more concentrated nanoparticle solution $\left(20 \mathrm{mg} \mathrm{mL}^{-1}\right.$ ) was deposited on top, and its distribution was immediately analysed.

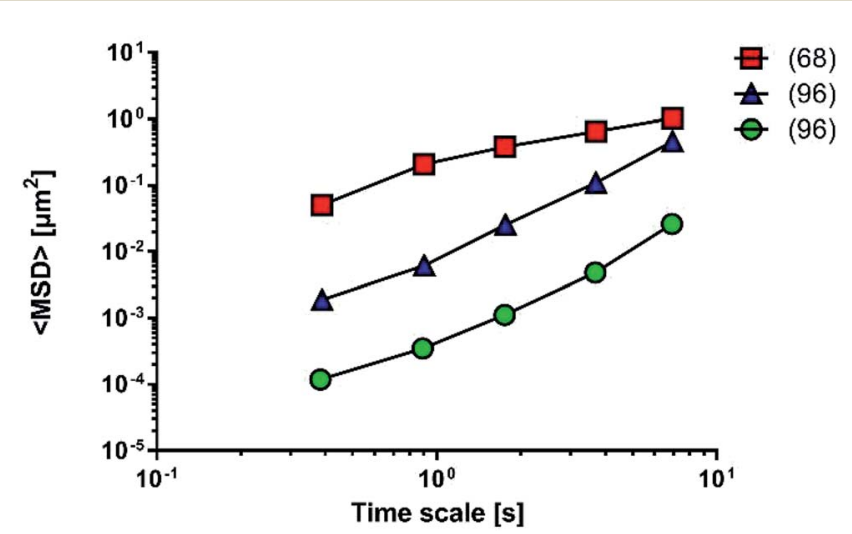

Fig. 10 Mean square displacement (MSD) of carboxylic nanoparticles across physiological mucus (red squares) or multi-component mucus model (blue triangles) and PLGA-DDAB/DNA nanoparticles across multi-component mucus model (green circles). The data were digitally extracted from the different sources. Each model is named after the reference number (numbers in brackets) and the type of sample. Before plotting, the data were homogenized in units of measurement to make them comparable. 
Also diffusion of poly(D,L-lactic-co-glycolic) acid (PLGA) and dimethyldioctadecylammonium bromide (DDAB) nanoparticles with condensed DNA on the surfaces was analysed in multicomponent models. ${ }^{96}$ Interestingly, their average transport rate was 10-fold higher than similar sized PS nanoparticles. Furthermore, PLGA-DDAB/DNA nanoparticles exhibited greater mobility than $\mathrm{COOH}-\mathrm{PS}$ ones. The difference in diffusion rate is probably related to the surface chemistry of the nanoparticles, as DNA confers a hydrophilic character to the particles that are repulsed by the hydrophobic mucin glycoproteins. It may easily interact with the more hydrophobic $\mathrm{COOH}-\mathrm{PS}$ nanoparticles, limiting their diffusion. Another possible explanation relies on the effect of each nanoparticle on the structure of the multicomponent model. Indeed, the authors hypothesise that aggregates of PLGA-DDAB/DNA nanoparticles may have reorganized the network structure inducing larger pores, thus leading to higher diffusion. In this way, PLGA-DDAB/DNA nanoparticles may represent a future tool for in situ delivery of genetic material or vaccines.

\section{Conclusions}

Several models mimicking some of the mucus properties, such as drug diffusion, permeability, viscosity and other viscoelastic characteristics, are available from the literature. They are usually based on mucin as a single component (monocomponents models) or combined with lipids and other proteins present in physiological mucus (multi-components models). However, the major limitation of these in vitro models is the lack of physical and biochemical anisotropy, which is a relevant intrinsic property of physiological mucus. Mucus is a selective barrier of biomolecules, such as nutrients and drugs, and simultaneously a suitable environment for microbial niches (while preventing epithelial infections). Hence, bioinspired in vitro mucus models represent perspective assets in drug screening/delivery research, regenerative medicine and unveil complex biological interactions as those involved in the gut-brain-axis. Despite this, mucus has been poorly interpreted in the literature as a material. A comparative analysis of the available data is an urgent need in the field of mucus modelling, as it provides proper and homogenized references - until now not proposed in this research area - for a further engineering of more advanced in vitro models and their potential application.

\section{Conflicts of interest}

There are no conflicts to declare.

\section{Acknowledgements}

This study was funded by the European Research Council (ERC) under the European Union's Horizon 2020 research and innovation program (Grant agreement No. 724734-MINERVA). The results reflect only the authors' views and the Agency is not responsible for any use that may be made of the information contained.

\section{References}

1 J. R. Kelly, C. Minuto, J. F. Cryan, G. Clarke and T. G. Dinan, Cross Talk: The Microbiota and Neurodevelopmental Disorders, Front. Neurosci., 2017, 11, 1-31.

2 S. Grenham, G. Clarke, J. F. Cryan and T. G. Dinan, BrainGut-Microbe Communication in Health and Disease, Front Physiol, 2011, 2, 1-15.

3 E. A. Mayer, R. Knight, S. K. Mazmanian, J. F. Cryan and K. Tillisch, Gut Microbes and the Brain: Paradigm Shift in Neuroscience, J. Neurosci., 2014, 34(46), 15490-15496.

4 N. Sudo, Y. Chida, Y. Aiba, J. Sonoda, N. Oyama, X.-N. Yu, et al., Postnatal microbial colonization programs the hypothalamic-pituitary-adrenal system for stress response in mice, J. Physiol., 2004, 558(1), 263-275.

5 G. Clarke, S. Grenham, P. Scully, P. Fitzgerald, R. D. Moloney, F. Shanahan, et al., The microbiome-gut-brain axis during early life regulates the hippocampal serotonergic system in a sex-dependent manner, Mol. Psychiatry, 2013, 18(6), 666673.

6 S. O. Reber, Stress and animal models of inflammatory bowel disease-An update on the role of the hypothalamopituitary-adrenal axis, Psychoneuroendocrinology, 2012, 37(1), 1-19.

7 P. P. Freestone, P. H. Williams, R. D. Haigh, A. F. Maggs, C. P. Neal and M. Lyte, Growth Stimulation of Intestinal Commensal Escherichia coli by Catecholamines: A Possible Contributory Factor in Trauma-Induced Sepsis, Shock, 2002, 18(5), 465-470.

8 ERC Funded Projects|ERC: European Research Council [Internet], 2019 [cited 2019 Jan 1], available from: http:// erc.europa.eu/erc-funded-projects.

9 I. Jakasa and S. Kezic, Evaluation of in vivo animal and in vitro models for prediction of dermal absorption in man, Hum. Exp. Toxicol., 2008, 27(4), 281-288.

10 M. T. Cronin, Non-animal approaches, the way forward, European Commission Scientific Conference, Brussels, 2017.

11 D. a. Fitts, Ethics and animal numbers: informal analyses, uncertain sample sizes, inefficient replications, and type I errors, J. Am. Assoc. Lab. Anim. Sci., 2011, 50(4), 445-453.

12 M. F. W. Festing and D. G. Altman, Guidelines for the Design and Statistical Analysis of Experiments Using Laboratory Animals, ILAR J., 2002, 43(4), 244-258.

13 UK Home Office, Annual Statistics of Scientific Procedures on Living Animals Great Britain 2017, National Statistics, 2018, pp. 1-33.

14 H. Buist, P. Craig, I. Dewhurst, S. Hougaard Bennekou, C. Kneuer, K. Machera, et al., Guidance on dermal absorption, EFSA J., 2017, 15(6), 1-60.

15 J. L. Parker and J. Clare Kohler, The success rate of new drug development in clinical trials: Crohn's disease, J. Pharm. Pharm. Sci., 2010, 13(2), 191-197.

16 Z. H. Shehadeh and M. S. Gordon, The role of the intestine in salinity adaptation of the rainbow trout, Salmo gairdneri, Comp. Biochem. Physiol., 1969, 30, 397-418. 
17 M. Mantle, G. Stewart, G. Zayas and M. King, The disulphidebond content and rheological properties of intestinal mucins from normal subjects and patients with cystic fibrosis, Biochem. J., 1990, 266(2), 597-604.

18 G. C. Hansson, Role of mucus layers in gut infection and inflammation, Curr. Opin. Microbiol., 2012, 15(1), 57-62.

19 L. A. Sellers, A. Allen, E. R. Morris and S. B. Ross-Murphy, The rheology of pig small intestinal and colonic mucus: weakening of gel structure by non-mucin components, Biochim. Biophys. Acta, Gen. Subj., 1991, 1115(2), 174-179.

20 M. G. Espey, Role of oxygen gradients in shaping redox relationships between the human intestine and its microbiota, Free Radic. Biol. Med., 2013, 55, 130-140.

21 J. He, L. Yi, L. Hai, L. Ming, W. Gao and R. Ji, Characterizing the bacterial microbiota in different gastrointestinal tract segments of the Bactrian camel, Sci. Rep., 2018, 8(1), 1-9.

22 M. E. V. Johansson, H. Sjövall and G. C. Hansson, The gastrointestinal mucus system in health and disease, Nat. Rev. Gastroenterol. Hepatol., 2013, 10(6), 352-361.

23 R. Okumura and K. Takeda, Maintenance of intestinal homeostasis by mucosal barriers, Inflamm. Regen., 2018, 38(1), 5 .

24 S. Macfarlane, H. Steed and G. T. Macfarlane, Intestinal bacteria and inflammatory bowel disease, Crit. Rev. Clin. Lab. Sci., 2009, 46(1), 25-54.

25 S. Tadesse, G. Corner, E. Dhima, M. Houston, C. Guha, L. Augenlicht, et al., MUC2 mucin deficiency alters inflammatory and metabolic pathways in the mouse intestinal mucosa, Oncotarget, 2017, 8(42), 71456-71470.

26 M. E. V. Johansson, M. Phillipson, J. Petersson, A. Velcich, L. Holm and G. C. Hansson, The inner of the two Muc2 mucin-dependent mucus layers in colon is devoid of bacteria, Proc. Natl. Acad. Sci. U. S. A., 2008, 105(39), 15064-15069.

27 R. D. Pullan, G. A. Thomas, M. Rhodes, R. G. Newcombe, G. T. Williams, A. Allen, et al., Thickness of adherent mucus gel on colonic mucosa in humans and its relevance to colitis, Gut, 1994, 35(3), 353-359.

28 H. Witas, J. Sarosiek, M. Aono, V. L. N. Murty, A. Slomiany and B. L. Slomiany, Lipids associated with rat smallintestinal mucus glycoprotein, Carbohydr. Res., 1983, 120, 67-76.

29 C. November, Diffusion coefficient in native mucus gel of rat small intestine, 1990, pp. 517-519.

30 A. W. Larhed, P. Artursson, J. Gråsjö and E. Björk, Diffusion of Drugs in Native and Purified Gastrointestinal Mucus, $J$. Pharm. Sci., 1997, 86(6), 660-665.

31 A. E. Bell, L. A. Sellers, A. Allen, W. J. Cunliffe, E. R. Morris and S. B. Ross-Murphy, Properties of Gastric and Duodenal Mucus: Effect of Proteolysis, Disulfide Reduction, Bile, Acid, Ethanol, and Hypertonicity on Mucus Gel Structure, Gastroenterology, 1985, 88(1), 269-280.

32 D. K. Podolsky and K. J. Isselbacher, Composition of human colonic mucin. Selective alteration in inflammatory bowel disease, J. Clin. Investig., 1983, 72(1), 142-153.

33 I. Röhe, F. J. Hüttner, J. Plendl, B. Drewes and J. Zentek, Comparison of different histological protocols for the preservation and quantification of the intestinal mucus layer in pigs, Eur. J. Histochem., 2018, 62(1), 2874.

34 S. K. Lai, Y.-Y. Wang, D. Wirtz and J. Hanes, Micro- and macrorheology of mucus, Adv. Drug Delivery Rev., 2009, 61(2), 86-100.

35 M. E. V. Johansson, J. M. H. Larsson and G. C. Hansson, The two mucus layers of colon are organized by the MUC2 mucin, whereas the outer layer is a legislator of hostmicrobial interactions, Proc. Natl. Acad. Sci. U. S. A., 2011, 108(suppl. 1), 4659-4665.

36 A. N. Round, N. M. Rigby, A. Garcia de la Torre, A. Macierzanka, E. N. C. Mills and A. R. Mackie, Lamellar Structures of MUC2-Rich Mucin: A Potential Role in Governing the Barrier and Lubricating Functions of Intestinal Mucus, Biomacromolecules, 2012, 13(10), 32533261.

37 A. Macierzanka, A. R. Mackie, B. H. Bajka, N. M. Rigby, F. Nau and D. Dupont, Transport of particles in intestinal mucus under simulated infant and adult physiological conditions: Impact of mucus structure and extracellular DNA, PLoS One, 2014, 9(4), 1-11.

38 H. Li, J. P. Limenitakis, T. Fuhrer, M. B. Geuking, M. A. Lawson, M. Wyss, et al., The outer mucus layer hosts a distinct intestinal microbial niche, Nat. Commun., 2015, 6, 8292.

39 M. E. V. Johansson, M. Phillipson, J. Petersson, A. Velcich, L. Holm, G. C. Hansson, et al., The inner of the two Muc2 mucin-dependent mucus layers in colon is devoid of bacteria, Proc. Natl. Acad. Sci. U. S. A., 2008, 105(39), 15064-15069.

40 M. Boegh, S. G. Baldursdóttir, A. Müllertz and H. M. Nielsen, Property profiling of biosimilar mucus in a novel mucuscontaining in vitro model for assessment of intestinal drug absorption, Eur. J. Pharm. Biopharm., 2014, 87(2), 227-235.

41 J. P. Pearson, P. I. Chater and M. D. Wilcox, The properties of the mucus barrier, a unique gel - how can nanoparticles cross it?, Ther. Deliv., 2016, 7(4), 229-244.

42 D. Ambort, S. van der Post, M. E. V. Johansson, J. MacKenzie, E. Thomsson, U. Krengel, et al., Function of the CysD domain of the gel-forming MUC2 mucin, Biochem. J., 2011, 436(1), 61-70.

43 D. Ambort, M. E. V. Johansson, J. K. Gustafsson, A. Ermund and G. C. Hansson, Perspectives on Mucus Properties and Formation-Lessons from the Biochemical World, Cold Spring Harbor Perspect. Med., 2012, 2(11), a014159.

44 L. Arike and G. C. Hansson, The Densely O-Glycosylated MUC2 Mucin Protects the Intestine and Provides Food for the Commensal Bacteria, J. Mol. Biol., 2016, 428(16), 32213229.

45 Y. S. Kim and S. B. Ho, Intestinal Goblet Cells and Mucins in Health and Disease: Recent Insights and Progress, 2010.

46 S. van der Post, D. B. Subramani, M. Bäckström, M. E. V. Johansson, M. B. Vester-Christensen, U. Mandel, et al., Site-specific O-Glycosylation on the MUC2 Mucin Protein Inhibits Cleavage by the Porphyromonas gingivalis Secreted Cysteine Protease (RgpB), J. Biol. Chem., 2013, 288(20), 14636-14646. 
47 A. Slomiany, S. Yano, B. L. Slomiany and G. B. J. Glass, Lipid composition of the gastric mucous barrier in the rat, J. Biol. Chem., 1978, 253(11), 3785-3791.

48 V. L. N. Murty, J. Sarosiek, A. Slomiany and B. L. Slomiany, Effect of lipids and proteins on the viscosity of gastric mucus glycoprotein, Biochem. Biophys. Res. Commun., 1984, 121(2), 521-529.

49 A. W. Larhed, P. Artursson and E. Björk, The influence of intestinal mucus components on the diffusion of drugs, Pharm. Res., 1998, 15(1), 66-71.

50 M. E. V. Johansson, K. A. Thomsson and G. C. Hansson, Proteomic analyses of the two mucus layers of the colon barrier reveal that their main component, the Muc2 mucin, is strongly bound to the fcgbp protein, J. Proteome Res., 2009, 8(7), 3549-3557.

51 C. L. Bevins and N. H. Salzman, Paneth cells, antimicrobial peptides and maintenance of intestinal homeostasis, Nat. Rev. Microbiol., 2011, 9(5), 356-368.

52 C. Dobson, Changes in the protein content of the serum and intestinal mucus of sheep with reference to the histology of the gut and immunological response to Oesophagostomum columbianum infections, Parasitology, 1967, 57(02), 201.

53 L. Thim, F. Madsen and S. S. Poulsen, Effect of trefoil factors on the viscoelastic properties of mucus gels, Eur. J. Clin. Investig., 2002, 32(7), 519-527.

54 H. Kindon, C. Pothoulakis, L. Thim, K. Lynch-Devaney and D. K. Podolsky, Trefoil peptide protection of intestinal epithelial barrier function: Cooperative interaction with mucin glycoprotein, Gastroenterology, 1995, 109(2), 516-523.

55 J. M. Wells, R. J. Brummer, M. Derrien, T. T. MacDonald, F. Troost, P. D. Cani, et al., Homeostasis of the gut barrier and potential biomarkers, Am. J. Physiol.: Gastrointest. Liver Physiol., 2017, 312(3), G171-G193.

56 U. Meyer-Hoffert, M. W. Hornef, B. Henriques-Normark, L.-G. Axelsson, T. Midtvedt, K. Putsep, et al., Secreted enteric antimicrobial activity localises to the mucus surface layer, Gut, 2008, 57(6), 764-771.

57 A. Lechanteur, J. das Neves and B. Sarmento, The role of mucus in cell-based models used to screen mucosal drug delivery, Adv. Drug Delivery Rev., 2018, 124, 50-63.

58 B. N. Lourenço, T. dos Santos, C. Oliveira, C. C. Barrias and P. L. Granja, Bioengineering a novel $3 \mathrm{D}$ in vitro model of gastric mucosa for stomach permeability studies, Acta Biomater., 2018, 82, 68-78.

59 C. R. Bakshani, A. L. Morales-Garcia, M. Althaus, M. D. Wilcox, J. P. Pearson, J. C. Bythell, et al., Evolutionary conservation of the antimicrobial function of mucus: a first defence against infection, npj Biofilms Microbiomes, 2018, 4(1), 14.

60 J. Y. Lock, T. L. Carlson and R. L. Carrier, Mucus models to evaluate the diffusion of drugs and particles, Adv. Drug Delivery Rev., 2017, 124, 34-49.

61 H. Lennernäs, Ö. Ahrenstedt, R. Hällgren, L. Knutson, M. Ryde and L. K. Paalzow, Regional Jejunal Perfusion, a New In Vivo Approach to Study Oral Drug Absorpion in Man, Pharm. Res., 1992, 09(10), 1243-1251.
62 A. L. Miller, H. D. R. Golledge and M. C. Leach, The Influence of Isoflurane Anaesthesia on Intestinal permeability in Healthy Dogs, PLoS One, 2016, 11(11), 117-123.

63 O. W. Meldrum, G. E. Yakubov, M. R. Bonilla, O. Deshmukh, M. A. McGuckin and M. J. Gidley, Mucin gel assembly is controlled by a collective action of non-mucin proteins, disulfide bridges, $\mathrm{Ca}^{2+}$-mediated links, and hydrogen bonding, Sci. Rep., 2018, 8(1), 1-16.

64 A. Macierzanka, N. M. Rigby, A. P. Corfield, N. Wellner, F. Böttger, E. N. C. Mills, et al., Adsorption of bile salts to particles allows penetration of intestinal mucus, Soft Matter, 2011, 7(18), 8077.

65 C. T. Nordgård and K. I. Draget, Dynamic responses in small intestinal mucus: Relevance for the maintenance of an intact barrier, Eur. J. Pharm. Biopharm., 2015, 95, 144-150.

66 L. A. Sellers, A. Allen, E. R. Morris and S. B. Ross-Murphy, Mechanical characterization and properties of gastrointestinal mucus gel, Biorheology, 1987, 24(6), 615623.

67 D. A. Norris and P. J. Sinko, Effect of size, surface charge, and hydrophobicity on the translocation of polystyrene microspheres through gastrointestinal mucin, J. Appl. Polym. Sci., 1997, 63(11), 1481-1492.

68 J. S. Crater and R. L. Carrier, Barrier Properties of Gastrointestinal Mucus to Nanoparticle Transport, Macromol. Biosci., 2010, 10(12), 1473-1483.

69 I. Osiecka, P. A. Porter, R. T. Borchardt, J. A. Fix and C. R. Gardner, In Vitro Drug Absorption Models. I. Brush Border Membrane Vesicles, Isolated Mucosal Cells and Everted Intestinal Rings: Characterization and Salicylate Accumulation, Pharm. Res., 1985, 2(6), 284-293.

70 P. S. Leppert and J. A. Fix, Use of Everted Intestinal Rings for In Vitro Examination of Oral Absorption Potential, J. Pharm. Sci., 1994, 83(7), 976-981.

71 M. A. Alam, F. I. Al-Jenoobi and A. M. Al-mohizea, Everted gut sac model as a tool in pharmaceutical research: limitations and applications, J. Pharm. Pharmacol., 2012, 64(3), 326-336.

72 I. J. Hidalgo, T. J. Raub and R. T. Borchardt, Characterization of the human colon carcinoma cell line (Caco-2) as a model system for intestinal epithelial permeability, Gastroenterology, 1989, 96(3), 736-749.

73 B. Sarmento, F. Andrade, S. B. da Silva, F. Rodrigues, J. das Neves and D. Ferreira, Cell-based in vitro models for predicting drug permeability, Expert Opin. Drug Metab. Toxicol., 2012, 8(5), 607-621.

74 N. Navabi, M. A. Mcguckin and S. K. Linde, Gastrointestinal Cell Lines Form Polarized Epithelia with an Adherent Mucus Layer when Cultured in Semi-Wet Interfaces with Mechanical Stimulation, PLoS One, 2013, 8(7), 1-15.

75 G. J. Mahler, M. L. Shuler and R. P. Glahn, Characterization of Caco-2 and HT29-MTX cocultures in an in vitro digestion/ cell culture model used to predict iron bioavailability, J. Nutr. Biochem., 2009, 20(7), 494-502.

76 A. Béduneau, C. Tempesta, S. Fimbel, Y. Pellequer, V. Jannin, F. Demarne, et al., A tunable Caco-2/HT29-MTX co-culture model mimicking variable permeabilities of the human 
intestine obtained by an original seeding procedure, Eur. $J$. Pharm. Biopharm., 2014, 87(2), 290-298.

77 F. Antunes, F. Andrade, F. Araújo, D. Ferreira and B. Sarmento, Establishment of a triple co-culture in vitro cell models to study intestinal absorption of peptide drugs, Eur. J. Pharm. Biopharm., 2013, 83(3), 427-435.

78 E. Gullberg, M. Leonard, J. Karlsson, A. M. Hopkins, D. Brayden, A. W. Baird, et al., Expression of Specific Markers and Particle Transport in a New Human Intestinal M-Cell Model, Biochem. Biophys. Res. Commun., 2000, 279(3), 808-813.

79 F. Araújo and B. Sarmento, Towards the characterization of an in vitro triple co-culture intestine cell model for permeability studies, Int. J. Pharm., 2013, 458(1), 128-134.

80 J. H. Sung, J. Yu, D. Luo, M. L. Shuler and J. C. March, Microscale 3-D hydrogel scaffold for biomimetic gastrointestinal (GI) tract model, Lab Chip, 2011, 11(3), 389-392.

81 J. Pusch, M. Votteler, S. Göhler, J. Engl, M. Hampel, H. Walles, et al., The physiological performance of a threedimensional model that mimics the microenvironment of the small intestine, Biomaterials, 2011, 32(30), 7469-7478.

82 H. Kimura, T. Yamamoto, H. Sakai, Y. Sakai and T. Fujii, An integrated microfluidic system for long-term perfusion culture and on-line monitoring of intestinal tissue models, Lab Chip, 2008, 8(5), 741.

83 H. Kitamura, M. Cho, B. H. Lee, J. R. Gum, B. B. Siddiki, S. B. Ho, et al., Alteration in mucin gene expression and biological properties of HT29 colon cancer cell subpopulations, Eur. J. Cancer, Part A, 1996, 32(10), 17881796.

84 T. Lesuffleur, N. Porchet, J. P. Aubert, D. Swallow, J. R. Gum, Y. S. Kim, et al., Differential expression of the human mucin genes MUC1 to MUC5 in relation to growth and differentiation of different mucus-secreting HT-29 cell subpopulations, J. Cell Sci., 1993, 106, 771-783.

85 S. Bhattacharjee, E. Mahon, S. M. Harrison, J. Mcgetrick, M. Muniyappa, S. D. Carrington, et al., Nanoparticle passage through porcine jejunal mucus: Microfluidics and rheology, Nanomed. Nanotechnol. Biol. Med., 2016, 13, 863873.

86 T. T. Kararli, Comparison of the gastrointestinal anatomy, physiology, and biochemistry of humans and commonly used laboratory animals, Biopharm Drug Dispos., 1995, 16(5), 351-380.
87 A.-C. Groo and F. Lagarce, Mucus models to evaluate nanomedicines for diffusion, Drug Discov. Today, 2014, 19(8), 1097-1108.

88 M. D. Wilcox, L. K. Van Rooij, P. I. Chater, I. Pereira De Sousa and J. P. Pearson, The effect of nanoparticle permeation on the bulk rheological properties of mucus from the small intestine, Eur. J. Pharm. Biopharm., 2015, 96, 484-487.

89 S. K. Lai, Y. Y. Wang, D. Wirtz and J. Hanes, Micro- and macrorheology of mucus, Adv. Drug Delivery Rev., 2009, 61(2), 86-100.

90 B. Mauroy, P. Flaud, D. Pelca, C. Fausser, J. Merckx and B. R. Mitchell, Toward the modeling of mucus draining from human lung: role of airways deformation on airmucus interaction, Front. Physiol., 2015, 6, 1-15.

91 S. Shak, D. J. Capon, R. Hellmiss, S. A. Marsters and C. L. Baker, Recombinant human DNase I reduces the viscosity of cystic fibrosis sputum, Proc. Natl. Acad. Sci. U. S. A., 1990, 87(23), 9188-9192.

92 M. D. Shub, K. Y. Pang, D. A. Swann and W. A. Walker, Agerelated changes in chemical composition and physical properties of mucus glycoproteins from rat small intestine, Biochem. J., 1983, 215(2), 405-411.

$93 \mathrm{~J}$. Moschera and W. Pigman, The isolation and characterization of rat sublingual mucus-glycoprotein, Carbohydr. Res., 1975, 40(1), 53-67.

94 M. De Salegui and H. Plonska, Preparation and properties of porcine submaxillary mucins, Arch. Biochem. Biophys., 1969, 129(1), 49-56.

95 J. Kočevar-Nared, J. Kristl and J. Šmid-Korbar, Comparative rheological investigation of crude gastric mucin and natural gastric mucus, Biomaterials, 1997, 18(9), 677-681.

96 M. Dawson, E. Krauland, D. Wirtz and J. Hanes, Transport of Polymeric Nanoparticle Gene Carriers in Gastric Mucus, Biotechnol. Prog., 2004, 20(3), 851-857.

97 D. L. Leiske, C. I. Leiske, D. R. Leiske, M. F. Toney, M. Senchyna, H. A. Ketelson, et al., Temperature-Induced Transitions in the Structure and Interfacial Rheology of Human Meibum, Biophys. J., 2012, 102(2), 369-376.

98 S. K. Sarna, Cyclic motor activity; migrating motor complex: 1985, Gastroenterology, 1985, 89(4), 894-913.

99 E. Deloose, P. Janssen, I. Depoortere and J. Tack, The migrating motor complex: control mechanisms and its role in health and disease, Nat. Rev. Gastroenterol. Hepatol., 2012, 9(5), 271-285. 